\title{
LABORATORIOS REMOTOS en la Formación por Competencias como instrumento didáctico en innovación educativa en asignaturas de carreras de Ingeniería

\author{
REMOTE LABORATORIES in Competency Training as a didactic instrument \\ in educational innovation in Engineering
}

\author{
Paula Andrea Zanetti \\ paula.zanetti@gmail.com \\ Ingeniería en Sistemas \\ UDEMM \\ Buenos Aires, Argentina
}

\begin{abstract}
Resumen - Existen muchas definiciones de lo que significa innovación e innovación educativa, pero lo que es necesario rescatar es que la innovación educativa tiene como objetivo primordial alcanzar la calidad de la educación en todos los niveles, especialmente en la Formación por Competencias. La necesidad detectada y que aborda este trabajo es por un lado, la formación de profesionales en Competencias tanto técnicas como sociales, y la propuesta de innovación educativa que las Instituciones Universitarias deben respaldar e implementar en sus carreras con todo el cambio organizacional, estratégico y operativo que esto conlleva. Los Laboratorios Remotos se pueden considerar una innovación educativa ya que le agregan instrumentación, control y acceso a equipos de laboratorio reales sumado a tener una adecuada formación general y por Competencias que les permita adquirir conocimientos y herramientas derivados del avance de la ciencia y de la tecnología. El Proyecto de una Red Argentina colaborativa de Laboratorios de acceso remoto potencia el desarrollo de ecosistemas de experimentación académica que invita a cada institución que forma parte de la Red se especialice en desarrollar una o un grupo de experiencias remotas que se ofrecerán y serán articuladas en un servicio Web.
\end{abstract}

Palabras clave: Laboratorios Remotos, Innovación educativa, Red colaborativa de Laboratorios didácticos, Formación en Competencias, experiencias remotas.

Abstract - There are many definitions of what educational innovation and innovation means, but the main focus is that educational innovation has as its main objective to achieve the quality of education at all levels, especially in Skills Training. The need detected and addressed by this work is, on the one hand, the training of professionals in both technical and social competencies, on the other hand, the educational innovation proposal that University Institutions must support and implement in their careers with all the organizational change, strategic and operational that this implies. Remote Laboratories can be considered an educational innovation since they add instrumentation, control and access to real laboratory equipment in addition to having an adequate general and Competency training that allows them to acquire knowledge and tools arising from the improvements in science and technology. The Project for a collaborative Argentine Network of Remote Access Laboratories promotes the development of academic experimentation ecosystems that invites each institution that is part of the Network to specialize in developing one or a group of remote experiences that will be offered and articulated in a Web service.

Keywords: Remote Laboratories, Educational Innovation, Collaborative Network of Didactic Laboratories, Training in Competencies, remote experiences.

\section{INTRODUCCIÓN}

La implementación del uso de los Laboratorios Remotos en carreras de grado de Ingeniería en formación por Competencias y la formación de una Red colaborativa de Laboratorios didácticos, favorece limitar la multiplicación de equipos similares en diferentes sedes permitiendo esto optimizar los recursos físicos, económicos y el capital humano. Pueden ser accedidos por estudiantes y docentes miembros de la red o no, alcanzando a estudiantes en todo el territorio nacional. Según Aranda et al. (2015), los laboratorios de acceso remoto están disponibles los 365 días del año y las $24 \mathrm{hs}$ del día extinguiendo límites institucionales, geográficos y temporales. Se puede acceder desde una tablet, teléfono celular, pc o notebook, teniendo disponibilidad en todo tipo de dispositivo. Las plataformas son de entorno amigable y muy simple de usar, habilitan el almacenamiento etiquetado y certificado de las experiencias. El estudiante observa y controla el desarrollo del experimento en su dispositivo de conexión en tiempo real y se pueden implementar en cualquier modalidad de E-learning. Basándose en el avance de los dispositivos móviles y el acceso a tecnologías web pueden abordar el proyecto para los estudiantes de ingeniería y proponer prácticas de laboratorio a distancia al alcance de todos.

Según Lorandi et al. (2011), se plantea que la realización de prácticas con equipo de laboratorio y prototipos didácticos es fundamental si se quiere consolidar los conceptos adquiridos en el aula y el autoaprendizaje. Además, las nuevas tecnologías basadas en Internet, la virtualización y la mejora tecnológica en servidores, pueden ser utilizadas para suplir la carencia de laboratorios y además enriquecer el desarrollo de prácticas en espacios y entornos virtuales con características innovadoras. 
Para implementar lo anteriormente expuesto, es necesario que las Instituciones aborden el tema del uso de los Laboratorios Remotos para la Formación en Competencias y además creen una comunidad colaborativa para ampliar, potenciar, actualizar y mejorar la propuesta entre Instituciones.

En estudios realizados por Santamaría Buitrago et al. (2016), se plantea potencializar el desarrollo de ecosistemas de experimentación académica, abiertos, reusables e interoperables, a través del diseño de marco de trabajo e integración tecnológica orientado a la implementación de laboratorios virtuales y teleoperados, permitiendo una alta escalabilidad y flexibilidad en la modificación del dimensionamiento del servicio apoyado en estándares, arquitecturas, tecnologías, herramientas y aplicaciones abiertas y de propósito general.

Y una vez establecido el problema a resolver, se trata de la mejora de la educación virtual en profesiones técnicas para competencias prácticas, y el analizar el entorno específico de los Laboratorios Remotos, teniendo en cuenta, (Ros, 2012):

- Seguridad de elementos, datos y personal,

- Posibilidad de que un experimento sea visto por muchas personas al mismo tiempo

- Riesgo bajo cuando el experimento es peligroso,

- Fácil acceso para personas discapacitadas.

\section{CONTEXTO}

\section{A. Laboratorios Remotos}

El Laboratorio Remoto se plantea como un instrumento didáctico que busca proporcionar al estudiante una forma rápida y eficaz de adquirir y aplicar conocimiento. Todo esto incluye una nueva filosofía basada en diseño, simulación, emulación e implementación contribuyendo a la formación de los ingenieros en competencias (Vega et al. 2002).

Para Lorandi et al. (2011), los Laboratorios Remotos se pueden considerar como una evolución de los Laboratorios Virtuales. En este caso al sistema computacional se le agregan instrumentación, control y acceso a equipos de laboratorio reales. Ya no hablamos de llevar a cabo prácticas en un simulador, sino que se trata de realizar actividades prácticas de forma local o remota a través de una Intranet o Internet, permitiendo la transferencia de información entre un proceso real y los estudiantes de manera unidireccional o bidireccional. Bajo este esquema el estudiante utiliza y controla los recursos disponibles en un laboratorio, mediante el uso de tarjetas de adquisición de datos, sensores e instrumentos de medida con interfaces de red y software como LabView ${ }^{1}$, por ejemplo. Según estos autores,la diferencia contra un Laboratorio Virtual estriba en las interfaces de hardware instaladas en el equipo real. Los Laboratorios Remotos presentan mayores ventajas que los Laboratorios Virtuales, debido a que los primeros proporcionan una interactividad con equipamiento real, en lugar de usar programas que simulan los procesos. Los Laboratorios Remotos son una innovación en el campo de la educación y debe prestarse atención tanto a su diseño, al estudio de las ventajas e inconvenientes, y sobre todo, a sus aportaciones didácticas. Las ventajas que pueden ofrecer son por ejemplo:

- Pueden aprovechar los Laboratorios Convencionales existentes, al integrar sistema computacional con equipo e instrumentos, aunque el ahorro no es el objetivo principal, ya que se requiere adquirir software que generalmente es costoso.

- A diferencia de los Laboratorios Virtuales, el estudiante no pierde la perspectiva real, ya que, por un lado, los instrumentos virtuales diseñados son idénticos a los reales $\mathrm{y}$, por otro, la respuesta de los sistemas es la de un sistema real y solo se usa simulación para comparar resultados.

- Se explotan las ventajas de una Intranet o de Internet, ya que se puede tener acceso al laboratorio a través de una red, permitiendo inclusive experimentación asíncrona y solo limitando el uso, un esquema de programación de tiempos de uso, ampliando la oferta de horarios para el estudiante en formación.

- Pueden convertirse en un recurso extremadamente enriquecedor en la formación y adquisición de Competencias, ofreciendo los resultados de la experimentación casi en tiempo real, sólo estando limitados por la latencia propia de la red.

- Es posible llevar a cabo experimentos de forma estructurada o incluso de manera libre, en la que el estudiante puede desarrollar habilidades para la solución de problemas, observación, interpretación y análisis de resultados, de forma similar a la que lo hacen sus profesores o los investigadores de su universidad.

- $\quad$ El acceso, al igual que en un Laboratorio Virtual, solo se limita a tener una computadora con un navegador de Internet.

- Los Laboratorios Remotos ofrecen a nuestros estudiantes, la posibilidad de controlar de forma remota las aplicaciones basadas en instrumentos virtuales, enfrentando los mismos ambientes que existen en las empresas que han tomado el camino de la automatización y la reingeniería de sus procesos.

Según Godoy et al. (2016), un estudio del estado del arte permite diferenciar dos tipos diferentes de laboratorios remotos según su aplicación:

1) Laboratorios remotos destinados a desarrollo e investigación.

2) Laboratorios remotos destinados a formación y entrenamiento de usuarios.

La diferencia más importante entre los mismos es el nivel de conocimientos requerido por parte del usuario acerca del equipo bajo prueba. Los usuarios de los Laboratorios Remotos destinados a investigación necesitan un alto nivel de conocimientos del equipo bajo prueba para poder utilizarlos, y permiten a los usuarios acceder a los recursos del laboratorio sin ninguna restricción. En los otros, los usuarios deben realizar experimentos con ayuda $\mathrm{u}$ orientación de otra persona, en

\footnotetext{
1 Software privativo de National Instruments que permite el diseño de instrumentación virtual con interfaces al mundo real mediante el uso de tarjetas y hardware de adquisición de datos
} 
nuestro caso, los docentes o jefes de laboratorio.

\section{B. Redes colaborativas de trabajo para innovación educativa}

En estudios realizados, como ser el de Santamaría Buitrago et al. (2016), mencionado anteriormente en el que se plantea potencializar el desarrollo de ecosistemas de experimentación académica permitiría generar Redes colaborativas entre Instituciones con carreras afines. Además estos autores agregan, que las tecnologías de la información y la comunicación (TIC), mantienen un desarrollo acelerado en las últimas décadas, modificando y potencializando diferentes ámbitos como la economía, la producción y la educación, entre otros, y a la vez demanda contar con personal con una formación de alto nivel. Así como realizar cambios estructurales en la educación formal, no formal e informal, y proporcionando nuevas características y principios en la enseñanza como el aprendizaje autónomo, autoformación, la desterritorialización, la descentración, la virtualización, la tecnologización y la sociabilidad virtual.

En este mismo trabajo de Santamaría Buitrago et al. (2016) y tomando como fuente a Cabrero (2008), se plantean algunas cuestiones de la Formación basada en Red:

- Permite que los estudiantes vayan a su propio ritmo de aprendizaje.

- Formación basada en el concepto de formación en el momento en que se necesita (just-in-time-training).

- Permite la combinación de diferentes materiales (auditivos, visuales y audiovisuales).

- Con una sola aplicación puede atenderse a un mayor número de estudiantes

- El conocimiento es un proceso activo de construcción

- Tiende a reducir el tiempo de formación de las personas

- Tiende a ser interactiva, tanto entre los participantes en el proceso (profesor y estudiantes) como con los contenidos

- Tiende a realizarse de forma individual, sin que ello signifique la renuncia a la realización de propuestas colaborativas.

- No siempre disponemos de los recursos estructurales y organizativos para su puesta en funcionamiento.

Conformar la Red colaborativa de laboratorios didácticos invita a desarrollar a las distintas Instituciones una o un grupo de experiencias remotas articuladas mediante un servicio en la Web. Para Pérez Tornero y Tejedor (2016), lo que llamamos innovación es un cambio mixto, parte inducido por circunstancias exteriores y parte provocado desde adentro. Sólo en la innovación auténtica el proyecto responde a una necesidad, a una demanda, a que solucione un problema o que constituya una estrategia consciente.

La búsqueda de aprendizajes transversales requiere nuevas formas de enseñar y aprender que superan los estrechos límites de las materias tradicionales, por lo que se concluye planteando la necesidad de desarrollar en el aula estrategias que posibiliten un trabajo docente colaborativo, un análisis reflexivo conjunto de los criterios de evaluación y de los estándares de aprendizaje, y un continuo intercambio de información sobre el proceso de aprendizaje del alumnado.

\section{Formación del egresado por Competencias}

Según el Libro Rojo del CONFEDI ${ }^{2}$ en su Marco Conceptual sobre los estándares de segunda generación para la acreditación de carreras de Ingeniería en la República Argentina infiere que La Práctica de la Ingeniería comprende el estudio de factibilidad técnico-económica, investigación, desarrollo e innovación, diseño, proyecto, modelación, construcción, pruebas, optimización, evaluación, gerenciamiento, dirección y operación de todo tipo de componentes, equipos, máquinas, instalaciones, edificios, obras civiles, sistemas y procesos. Las cuestiones relativas a la seguridad y la preservación del medio ambiente constituyen aspectos fundamentales que la práctica de la ingeniería debe observar. La definición de Ingeniería y Práctica de la Ingeniería brindan la descripción conceptual de las características del graduado y constituyen la base para el análisis de las cuestiones atinentes a su formación. Esto lleva a la necesidad de proponer un currículo con un balance equilibrado de competencias y conocimientos académicos, científicos, tecnológicos y de gestión, con formación humanística. Los graduados de carreras de ingeniería deben tener una adecuada formación general, que les permita adquirir los nuevos conocimientos y herramientas derivados del avance de la ciencia y tecnología.

Gutiérrez Pallares et al. (2020), expresan en su artículo un aporte a la visión general de los aspectos más relevantes de un modelo de $\mathrm{EaD}$ que por medio de la consideración de componentes iniciales fundamentales en el tipo de educación se busca complementar y dar solución a los pilares de la educación tradicional, factores fundamentales como la presencialidad y la distancia deben ser acotados por la transformación de modelos que dan una respuesta a los paradigmas de la nueva educación.

\section{DESCRIPCIÓN}

\section{A. Innovación Curricular de las carreras en Ingeniería}

El estudio de la implementación del uso de Laboratorios Remotos (LR) en asignaturas de Ingeniería para la Formación de egresados en Competencias por un Modelo de Formación por Competencias según el Libro Rojo ${ }^{3}$ de CONFEDI del 2018, que propone para las carreras de ingeniería el aseguramiento de la calidad, y la definición de un nuevo estándar nacional para el tercer ciclo de acreditación obligatoria, fundamentada en los siguientes objetivos:

\section{- Actualizar y consolidar el actual modelo de formación}

${ }^{2}$ Consejo Federal de Decanos de Ingeniería de la República Argentina

3 propuesta de estándares de segunda generación para la acreditación de carreras de ingeniería en la república argentina - aprobado por la 63 Asamblea del CONFEDI de la República Argentina (Rosario, 1 de junio de 2018). Y presentado como propuesta ante el Ministerio de Educación, el Consejo Interuniversitario Nacional y el Consejo de Rectores de Universidades Privadas. 
de ingenieros.

- Consolidar un modelo de aprendizaje centrado en el estudiante.

- Definir un modelo comparable internacionalmente.

- Definir un enfoque basado en competencias y descriptores de conocimiento.

- Asegurar el cumplimiento de las actividades reservadas definidas para cada título.

\section{B. Los Laboratorios Remotos como innovación tecnopedagógica}

Los Laboratorios Remotos (LR) son una innovación en el campo de la educación y debe prestarse atención tanto a su diseño, al estudio de las ventajas e inconvenientes, y sobre todo, a sus aportaciones didácticas. Además se deberán contemplar algunas dificultades para el diseño de las propuestas didácticas, como ser: la experimentación en tiempo real exige períodos de muestreo relativamente pequeños, es necesario implementar protocolos de comunicaciones y esquemas de seguridad, para poder manipular los sistemas e interactuar con el hardware existente es necesario utilizar entradas y salidas digitales o analógicas, y por último, y muy importante, considerar los elevados costos, la escasa formación del profesorado en aspectos de instrumentación virtual especializada, la falta de recursos informáticos y la carencia de un área de desarrollo de software de apoyo académico institucional que supone desarrollar estos sistemas. La incorporación de las TIC en las universidades ayuda a resolver el problema de la demanda educativa. Según el artículo de la Revista de Ciencias Sociales y Humanidades Chakiñan, diciembre 2020 expone, que en definitiva, para poder ofertar y asegurar pertinencia de la educación, debe mostrarse una articulación coherente y armónica de un modelo que, por encima de los instrumentos, privilegie el sentido pedagógico de los procesos entendiendo que según (Mestre y Ruíz 2013:19) una educación de calidad puede salir adelante con una tecnología inadecuada; pero jamás una tecnología excelente podrá sacar adelante un proceso educativo de baja calidad.

\section{Infraestructura de apoyo en la implementación de la innovación}

La selección del LMS para definir la interfaz de usuario que cumpla con los requisitos definidos se determina con un sistema para la gestión del aprendizaje LMS que permita la gestión y la interacción con la plataforma (Santamaría Buitrago et al. 2016). Para ello se parte de la caracterización, selección y elección del sistema informático a utilizar, esta herramienta deberá recibir las peticiones del usuario, ya sea este el administrador, profesor, estudiante o invitado, y el consumo de los servicios y herramientas de aprendizaje necesarias para cada uno de los roles. Para cumplir con sus funciones el sistema de gestión de aprendizaje deberá cumplir con las siguientes funciones:

- Brindar un acceso seguro,

- Permitir interacción,

- Brindar en un entorno intuitivo la navegación dentro del portal,
- Diversidad de recursos y herramientas para la formación y comunicación entre los usuarios,

- Interoperabilidad de herramientas,

- Acceso a la información y contenidos proporcionado diversidad de objetos virtuales de aprendizaje y contenidos compatibles,

- Administración sencilla que permita las actividades relacionadas con la gestión académica, como registro, consulta de perfiles y registros, de una manera directa y sencilla,

- Ofrecer la posibilidad de trabajo colaborativo entre usuarios a través de aplicaciones que permitan compartir información y trabajar con documentos conjuntos,

- Seguimiento del rendimiento del estudiante que informen al docente de la participación del alumno y sobre el avance en las actividades académicas programadas y los resultados de la evaluación si se requiere, y

- Debe ser de carácter libre y de código abierto, requerido para la integración de los diferentes componentes de la plataforma.

\section{Redes de trabajo colaborativo}

Además, si se suma la formación de una Red colaborativa de Laboratorios didácticos entre Instituciones con carreras afines implicando una innovación tecnológica y educativa en las Instituciones que participen a través de una plataforma de web de gestión y administración de los experimentos. Los aspectos claves para organizar esta Red serían: relevar el estado actual de implementación en las distintas unidades educativas del país, invitar a todas las instituciones que quieran participar, y que todas las instituciones ofrezcan a la RED experiencias remotas que se puedan articular en la plataforma.

Para todo esto, se desarrollará una plataforma de web de gestión y administración de los experimentos. Se pretende la creación de un protocolo estándar que permita facilitar a la Red colaborativa continuar sumando experimentos que hagan crecer a la plataforma que tiene como objetivo principal un fin pedagógico. Por otra parte, al tratarse de una plataforma abierta se sientan las bases para que alumnos de las distintas Universidades que trabajen en proyectos de investigación o Proyectos finales de carrera y externos que formen parte de la Red puedan seguir agregando e integrando funcionalidades a la plataforma y así seguir creciendo en nuevas formas de aprendizaje.

Para esto se deberá realizar un relevamiento de todas las experiencias actuales en las distintas unidades académicas que formen parte de la Red, generar equipos autónomos de trabajo y, que cada Institución forme parte de Laboratorios NODOS de la Red. Cuantos más NODOS interconectados existan, más temas se pueden abarcar y ofrecer a los estudiantes. Los Laboratorios Remotos son plataformas (Godoy et al. 2016) que permiten el acceso remoto a diferentes tipos de equipos, dispositivos, laboratorios, etc. a través de Internet. Son sistemas complejos que incluyen un gran número de componentes. Están compuestos principalmente por dos sistemas: el sistema bajo prueba (equipamiento, máquinas eléctricas, dispositivos electrónicos, un laboratorio físico, etc.) 
y, el sistema de acceso remoto, que permite a los usuarios acceder al sistema bajo prueba para realizar experimentos remotamente. El sistema de acceso remoto está formado por diferentes módulos de hardware y software, entre estos:

- Sistema de interconexión (entre el sistema bajo prueba y el resto del laboratorio remoto).

- Autentificación de usuarios.

- Sistema de reserva de turnos.

- Base de datos.

- Interface web.

Según el artículo de Lòpez Luro et al. (2009) se puede presentar, como lo muestra la Figura 1, una arquitectura general donde se enfoca en los Clientes de laboratorios y en el concepto y la operación de lo que llamamos Distribuidor y Manejador de Conexiones (DMC) y Concentrador de Conexiones (CDC). Estos últimos, proveen a los Servidores de laboratorios la funcionalidad necesaria para poder gestionar distintos laboratorios al mismo tiempo, permitir el acceso a grupos de alumnos por laboratorio y conectarse para trabajar de manera concurrente y colaborativa, permitiendo opcionalmente, la presencia de un facilitador que altere la dinámica de trabajo durante la práctica en forma remota, cómo la indica la siguiente figura, y complementa dichas tecnologías y aplicaciones instauradas en la comunidad de usuarios de Internet, permitiendo el acceso a recursos de variada naturaleza, a través de interfaces bien definidas:

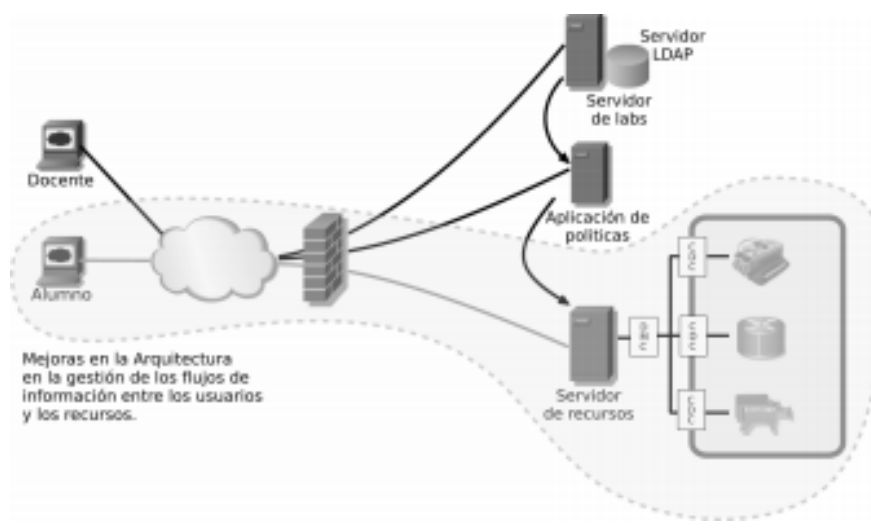

Figura 1

En las tecnologías emergentes, el desarrollo de materiales curriculares y unidades didácticas en las instituciones académicas en sistemas escalables de Cloud Computing requiere de una orquestación de un grupo de investigación centrado en la comunicación, colaboración e integración entre desarrolladores de software y los profesionales de operaciones en las tecnologías de la información (Santamaría Buitrago et al. 2016).

\section{E. Caso real de implementación}

Considerando este planteamiento como base, se observa que en realidad el desarrollo de Laboratorios Remotos es una herramienta poderosa en la construcción de habilidades instrumentales en carreras tecnológicas como la electricidad o la electrónica, y no se limita al ámbito académico universitario, también puede ser utilizado en la formación y cualificación del personal de las empresas, o en educación media y profesional (Cárdenas Gutiérrez et al. 2017). Tener infraestructura de laboratorios remotos tiene una gran ventaja sobre los laboratorios presenciales, porque permite desarrollar un modelo de Infraestructura como Servicio (IaaS), maximizando así el tiempo de uso en los lapsos donde los laboratorios tradicionales están fuera de uso, por ejemplo, horario no laboral, vacaciones, fines de semana, etc. De esta manera, las instituciones educativas pueden tener ingresos adicionales sobre su infraestructura.

Una implementación que se está realizando en una Universidad en la ciudad de Buenos Aires, Argentina propone que se realice de la siguiente forma: alumnos que egresan de la carrera de Ingeniería en Sistemas y de Ingeniería Electrónica se mancomunaron mediante el desarrollo de sus respectivos Proyectos Finales de egreso y de ambos Proyectos se desprenden dos aristas, la primera, es la creación de un equipo mecánico que pueda ejecutar un experimento, en este caso de Física (Ingeniería Electrónica) y la segunda, el diseño e implementación de una plataforma de administración de los Laboratorios Remotos, basado en la figural (Ingeniería en Sistemas). Basados en el avance de los dispositivos móviles y el acceso a tecnologías web se puede encarar este proyecto que será de importancia en las prácticas de laboratorio para los estudiantes de Ingeniería. En la era On Demand estar a la altura de proponer prácticas de laboratorio a distancia al alcance de todos mediante el uso de la tecnología disponible al alcance de todos.

\section{Resultados}

El potencial más fuerte que tiene este tipo de proyecto es poder llevar el alcance a otro nivel, un nivel de integración con otras entidades, educativas o con fines relacionados a la ciencia, que deseen poner a disposición sus equipos de laboratorio para experimentos y que estudiantes de todo el mundo puedan acceder a estas experiencias. El objetivo secundario que propone es la creación de un protocolo estándar que permita formar una red colaborativa que continúe sumando experimentos que hagan crecer a la plataforma que tiene como objetivo principal un fin pedagógico basado en Competencias profesionales.

De los resultados obtenidos se demuestra la viabilidad de la convergencia y representa un referente para el desarrollo de experimentación Cloud Computing abierta, extensible, interoperable, eficiente, escalable y a bajo costo en ambientes de experimentación académica. Siendo las tecnologías y/o aplicaciones emergentes, las que han probado su validez en ámbitos comerciales y académicos. El problema se centra en las instituciones académicas porque tendrán una tarea primordial en el desarrollo de materiales curriculares y unidades didácticas para la impartición en sistemas escalables de Cloud Computing. La orquestación de un grupo de investigación centrado en la comunicación, colaboración e integración entre desarrolladores de software y profesionales de operaciones en las tecnologías de la información de diferentes instituciones académicas, aprovecha de manera óptima los recursos hardware con los que se dispone.

Para Aranda et al. (2015), los estudios universitarios de carácter científico-técnico son buenos candidatos para implementar innovaciones educativas relacionadas con las TIC. 
Los estudiantes de estas disciplinas están habituados al uso de las TIC, por lo que pueden adoptar rápidamente nuevas tecnologías para el aprendizaje en Competencias. Además, muchas de las asignaturas científico-técnicas requieren la realización de prácticas de laboratorio, cuya implementación a través de plataformas virtuales de enseñanza constituye un sugerente reto, y a la vez una gran oportunidad, para el mundo académico.

\section{CONCLUSIONES}

En los desafíos de la Educación Superior en la República Argentina está proponer egresados de Ingeniería desde la visión de un ser competente. Esto significa que hayan desarrollado un conjunto de competencias que lo hacen capaz de ejercer su profesión en la realidad que los rodea. Todo esto significa un cambio de paradigma educativo, centrado en el aprendizaje, la innovación tecnológica, las competencias a desarrollar, los contenidos impartidos y el diseño de las propuestas didácticas. A nivel institucional contemplar las nuevas Actividades reservadas propuestas por el Libro Rojo, la acreditación de actividades y competencias, la virtualidad en el proceso de enseñanza y aprendizaje, el acceso a laboratorios remotos y de simulación, equiparar los sistemas de acreditación con los modelos internacionales, aumentar la tasa de graduados, el reconocimiento de las actividades de desarrollo tecnológico con impacto social.

Todo lo que implica el pasaje a un modelo centrado en el estudiante en la formación por competencias involucran a la docencia, a la investigación, extensión y gestión universitaria. Innovar en Educación en Ingeniería es un importante desafío para marcar líneas conceptuales y metodológicas tendientes a mejorar la calidad de las carreras de Ingeniería. Todo lo anteriormente expuesto demandará un proceso complejo de reflexión, reconfiguración, cambios de fondo que no sabemos si todas las instituciones superiores están dispuestas a transitar.

La idea de acceder a prácticas de formación experimental en forma remota comienza a tomar impulso en un reducido número de unidades académicas de la Argentina. Acceder en forma remota a los equipos instalados en un laboratorio universitario para operarlos e interactuar con ellos en actividades formativas, donde el estudiante tiene que contextualizar el experimento, seleccionar y parametrizar equipos, obtener resultados, calcular incertidumbres y sacar conclusiones; resulta un desafío enorme, que excede la implementación de simulaciones basadas en software. Al mismo tiempo, los Laboratorios de Acceso Remoto organizados en forma de Red, se presentan como una herramienta fuertemente inclusiva, ya que permiten a estudiantes de todas las regiones del país acceder a un surtido catálogo de las mejores prácticas de formación experimental, sin las limitaciones que impone la presencialidad y la pertenencia a una $u$ otra institución. Los aspectos claves para organizar esta Red serían: relevar el estado actual de implementación en las distintas unidades educativas del país, invitar a todas las instituciones que quieran participar, y que todas las instituciones ofrezcan a la RED experiencias remotas que se puedan articular en la plataforma.

\section{REFERENCIAS}

Aranda, J. G., Novakova, J., Rivilla, A. M., \& San Juan, C. R. (2015). Validación de requisitos funcionales de un Laboratorio Virtual Remoto como apoyo al blended learning. RED - Revista de Educación a Distancia, 45, 137. https://doi.org/10.6018/red/45/gamo

Cárdenas Gutierréz, C., Farías Martínez, G. y Méndez Castro, G. (2017). ¿Existe Relación entre la Gestión Administrativa y la Innovación Educativa? Un Estudio de Caso en Educación Superior. REICE., 15(1), 19-35. Recuperado https://revistas.uam.es/reice/article/view/6960.

Godoy, P., Cayssials, R. Y García Garino, C. (2016). Laboratorio Remoto para la Formación de Usuarios Basado en el Cloud. Revista: TE \& ET; no. 18. Páginas: 7-18. Facultad de Informática. ISSN: 1850-9959. Recuperado http://sedici.unlp.edu.ar/handle/10915/58478

Gutierréz Pallares, E., Rámirez Sánchez, M. y Borges Gouveia, L. (2020). Construcción de un modelo educativo a distancia con factores de aprendizaje y plataformas tecnológicas. Chakiñan, revista de ciencias sociales y humanidades,(12),18-31. https://doi.org/10.37135/chk.002.12.01

Lòpez Luro, F., Bertogna, M., Sánchez, L., Rodríguez, J. y Castillo, R. (2009). Infraestructura para laboratorios de acceso remoto. Revista: TE \& ET; no. 4. Facultad de Informática. Recuperado de http://sedici.unlp.edu.ar/handle/10915/14186.

Lorandi, A., Hermida, G., Hernández, J., \& Ladrón de Guevara, E. (2011). Los Laboratorios Virtuales y Laboratorios Remotos en la Enseñanza de la Ingeniería. Revista Internacional de Educación En Ingeniería, 4, 24-30. Recuperado http://bibliografia.eovirtual.com/LorandiA 2011 Labora torios.pdf

Libro Rojo de CONFEDI (2018). Propuestas de estándares de segunda generación para la acreditación de carreras de ingeniería en la RA. Disponible en: https://confedi.org.ar/download/documentos_confedi/LI BRO-ROJO-DE-CONFEDI Estandares-de-SegundaGeneracion-para-Ingenieria-2018.

Rodriguez Lestegás, F., Macía Arce, X. y Armas Quintá, F.(2020). De los contenidos a las competencias: aprender transversalmente a través del conocimiento social. Artículo realizado en el marco del proyecto de investigación PGC2018-094491-B-C31. Convocatoria 2018 del Plan Nacional de I+D+i y Fondos FEDER. https://doi.org/10.15304/ie.30.6944

Santamaría Buitrago, F. A., Ballesteros Ricaurte, J. A., \& González Sanabria, J. S. (2016). Plataforma Cloud Computing Como Infraestructura Tecnológica Para Laboratorios Virtuales, Remotos Y Adaptativos. Revista Científica, 3(23), 98. Retrieved from http://revistas.udistrital.edu.co/ojs/index.php/revcie/articl e/view/986. 